\title{
Association Between Endometriosis and Preterm Birth in Women With Spontaneous Conception or Using Assisted Reproductive Technology: A Systematic Review and Meta-Analysis of Cohort Studies(Review)
}

- Pérez-López, F.R.abEmail Author,

- Villagrasa-Boli, P.a,

- Muñoz-Olarte, M.a,

- Morera-Grau, Á. ${ }^{a}$,

- Cruz-Andrés, P.a,

- Hernandez, A.V.cd

$\bullet$

- View Correspondence (jump link)

- $\quad$ aFacultad de Medicina, Universidad de Zaragoza, Red de Investigación en Ginecología, Obstetricia y Reproducción, Instituto Aragonés de Ciencias de la Salud (IACS), Zaragoza, Spain

- 'DDepartment of Obstetrics and Gynecology, Lozano Blesa University Hospital, Zaragoza, Spain

- 'School of Medicine, Universidad Peruana de Ciencias Aplicadas (UPC), Lima, Peru

View additional affiliations

Abstract_View references (47)

Objective: To perform a systematic review and meta-analysis to estimate the effect of endometriosis on preterm birth (PB) risk. Methods: Searches were conducted in PubMed-MEDLINE, Embase, Scopus, Web of Science, Cochrane Library, Google Scholar, and SciELO for studies published in all languages from inception through April 2017. We included cohort studies evaluating pregnant women with and without endometriosis and conception either by spontaneous conception (SC) or with assisted reproductive technology (ART). Primary outcome was PB $(<37$ weeks), and secondary outcomes were intrauterine growth restriction (IUGR), low birthweight, small for gestational age (SGA), and birthweight. Pooled odds ratios (ORs) and its 95\% confidence interval (Cl) were calculated as effects, and randomeffects models were used for meta-analyses. Risk of bias was assessed with the Newcastle-Ottawa Scale, and heterogeneity of effects among studies was described with the I' statistic. Results: We identified 9 cohort studies including a total of 1496 715 pregnancies (13 798 with endometriosis diagnosis). In women with endometriosis, the PB risk was significantly increased in both SC (OR: $1.59 ; 95 \% \mathrm{Cl}$ : 1.32-1.90) and ART (OR: 1.43; 95\% Cl: 1.14-1.79). The SGA risk was increased in women with endometriosis (OR: 1.16; 95\% Cl: 1.05-1.28), while the IUGR and low birthweight risks and birthweight were not affected by endometriosis. Conclusion: Endometriosis is associated with increased PB risk in both SC and 
women who obtained pregnancy using ART. Prospective studies evaluating relevant outcomes are needed to confirm these results. (c) 2018, (C) The Author(s) 2018.

Author keywords

- assisted reproductive technology

- birthweight

- endometriosis

- infertility

- meta-analysis

- preterm birth

- small for gestational age

- spontaneous conception

Indexed keywords

EMTREE medical terms: birth weightcohort analysisconceptiondisease associationendometriosisfemalege agehumaninfertilityinfertility therapyintrauterine growth retardationlow birth we Ottawa scalepregnancy outcomepregnant womanpremature laborpriority journal infantsystematic review

- ISSN: 19337191

- Source Type: Journal

- Original language: English

- DOI: $10.1177 / 1933719117749760$

- Document Type: Review

- Publisher: SAGE Publications Inc. 